Recepción: 20 / 10 / 2017

Aceptación: 9 / 11 / 2017

Publicación: 15 / 12 / 2017
Ciencias Administrativas

Artículo Científico

\title{
El comportamiento organizacional en su entorno hacia la actitud y conducta del ser humano para lograr ventaja competitiva en el desempeño laboral
}

The organizational behavior in their environment towards the attitude and behavior of the human being to achieve a competitive advantage in the work performance

\section{Comportamento organizacional em seu ambiente em relação à atitude e comportamento do ser humano para obter vantagem competitiva no desempenho no trabalho}

Luz Teresa Cañarte Quimis ${ }^{\mathrm{I}}$

luztere2006@hotmail.com

Cristhian Yamir Moreira Cañarte II

cymcazulyplomo@hotmail.com
Miguel Augusto Baque Cantos ${ }^{\text {III }}$ miguel.baque@unesum.edu.ec

Mariana de Lourdes Cantos Figueroa ${ }^{\text {IV }}$ mariana.cantos@unesum.edu.ec

Correspondencia: luz.canarte@unesum.edu.ec

I. Master en Docencia Mención: Gestión Desarrollo del Currículo; Diplomado Internacional “Autoevaluación y Acreditación Universitaria" (Universidad Aconcagua, Republica de Chile); Docente Principal Titular de la Universidad Estatal del Sur de Manabí, Jipijapa, Ecuador.

II. Ingeniero Comercial. Mención Comercio Exterior Universidad Estatal del Sur de Manabí, Jipijapa, Ecuador.

III. Ingeniero Comercial: Universidad Técnica de Manabí; Diplomado en Autoevaluación y Acreditación Universitaria. Universidad Aconcagua República de Chile; Magister. Docencia Universitaria e Investigación Educativa. Universidad Nacional de Loja; Docente principal titular Universidad Estatal del Sur de Manabí, Jipijapa, Ecuador.

IV. Ingeniera Comercial de la Universidad Técnica de Manabí; Diplomado en autoevaluación y acreditación universitaria de la Universidad Aconcagua de la República de Chile; Magister en Docencia universitaria e investigación educativa Universidad Nacional de Loja; Docente principal titular Universidad Estatal del Sur de Manabí, Jipijapa, Ecuador. 
El comportamiento organizacional en su entorno hacia la actitud y conducta del ser humano para lograr ventaja competitiva en el desempeño laboral

\section{Resumen}

Los procesos motivacionales son importantes en los entornos laborales de las empresas, entendiendo que el trabajador es el alma de la misma, y que influye directamente en su productividad y crecimiento. Es por ello que es importante generar todas las herramientas necesarias para crear las condiciones laborales idóneas para que el trabajador se sienta a gusto en su entorno y se generen los resultados propuestos que no es más que crear ambientes laborales en armonía y que se reconozca el valor y talento humano que tienen los trabajadores dentro de las organizaciones, para ello es indispensable la utilización de herramientas importantes como la gestión por competencias y procesos de coaching empresariales que permitan determinar la mejor manera de cómo llevar la administración y gerencia de una organización entorno a su capital humano. La metodología utilizada en la investigación es de tipo documental no experimental. Algunos de los resultados que se pueden mencionar es que un clima organizacional propicio incrementará las iniciativas para promover nuevos negocios, acometer nuevos proyectos y solucionar problemas; mejorará la comunicación interna; aumentará la competitividad y facilitará el gobierno de la organización. Y entre las conclusiones más relevantes es que para que las organizaciones puedan lograr un alto grado de eficiencia es necesario trabajar en ambientes sumamente motivadores, participativos y con un personal altamente motivado e identificado con la organización.

Palabras claves: Organización, trabajador, motivación, entorno, eficiencia. 


\begin{abstract}
Motivational processes are important in the work environments of companies, understanding that the worker is the soul of the same, and that directly influences their productivity and growth. That is why it is important to generate all the necessary tools to create the ideal working conditions for the worker to feel comfortable in their environment and generate the proposed results that is nothing more than creating work environments in harmony and that the value is recognized and human talent that workers have within organizations, for this it is essential to use important tools such as management skills and business coaching processes to determine the best way to take the administration and management of an organization around their human capital. The methodology used in the research is non-experimental documentary type. Some of the results that can be mentioned is that an enabling organizational climate will increase the initiatives to promote new businesses, undertake new projects and solve problems; improve internal communication; It will increase competitiveness and facilitate the governance of the organization. And among the most relevant conclusions is that in order for organizations to achieve a high degree of efficiency it is necessary to work in highly motivating, participatory environments with a highly motivated and identified staff with the organization.
\end{abstract}

Key words: Organization, worker, motivation, environment, efficiency. 


\section{Introducción.}

Hoy en día debido a los procesos de cambios y las nuevas tecnologías que surgen en el mercado y que determinan el desarrollo de más habilidades, destrezas y conocimientos, las organizaciones se han visto en la necesidad de implementar cambios en su estrategia laboral a la hora de enfrentar los retos que se les presentan Si bien las organizaciones actualmente se encuentran afectadas por una crisis de índole económico, político, social, cultural, entre otros. Así como, elementos externos que afectan los procesos organizacionales y gerenciales. En este sentido, se hace necesario, que las empresas desarrollen nuevas técnicas de producción, mercado, distribución, servicio y atención al cliente, lo cual necesariamente amerita de la calidad del talento humano, para enfrentar con una buena y rápida capacidad de respuesta los retos organizacionales (Quintero, Africano, \& Faría, 2008). Esto es muy importante, ya que al tener un entorno laboral estable dentro de una organización, permite que los niveles de productividad aumenten, de esto hay muchos ejemplos (bonos de producción, empleados del mes, capacitación continua y permanente, aspectos motivacionales, entre otros). Hay empresas que han entendido estos temas y se esfuerzan día a día para aplicar las diferentes metodologías existentes.

Una organización laboral puede verse como un micro-ambiente, un subconjunto abierto limitado en el espacio y el tiempo, compuesto por individuos, puestos y áreas de trabajo, sus actividades y una variedad de elementos, tanto del medio físico o natural como de carácter cultural (Salazar, Guerrero, Machado, \& Cañedo, 2009). Las empresas son pequeñas ciudades, donde se crean relaciones laborales, personales, se adaptan al entorno en donde se establecen e incluso muchas cambian el ritmo de vida de los entornos en donde se desarrollan. 
En este sentido, las organizaciones en la actualidad deben diseñar y aplicar sistemas de evaluación del desempeño que les permitan identificar de manera oportuna y eficaz las fallas y limitaciones que se registran para corregirlas y garantizar el alcance de los objetivos institucionales. En este contexto, hay que considerar al respecto que uno de los principales factores que incide en el rendimiento laboral es la remuneración que los trabajadores perciben a cambio de su labor, junto a la estabilidad laboral que ofrece la empresa. Por ello, se persigue la creación de un ambiente favorable para el ejercicio y la estabilidad laboral, mediante la negociación y aprobación de contratos colectivos ajustados a las normas legales existentes y que incorporen salarios dignos que satisfagan a los trabajadores y que repercutan en sus rendimientos. Por lo tanto, se hace necesario precisar el desempeño del personal contratado, la estabilidad laboral que ofrece la organización y la relación entre ambas variables como parte de un proceso de evaluación y consolidación del funcionamiento de las organizaciones (Pedraza, Amaya, \& Conde, 2010). Como se mencionó anteriormente uno de los mayores incentivos que pueden tener los trabajadores de una empresa es el aspecto salarial, los bonos de productividad, las horas extras, los reconocimientos de empleados del mes, generan una motivación extra en el trabajador que hace que se comprometa aún más con su empresa y se genere un sentido de pertenecía. Hay casos donde existen competencias entre trabajadores para destacarse y ser cada día mejor.

Por otra parte, a pesar que el estudio del clima organizacional se encuentra enfocado a la comprensión de las variables ambientales internas que afectan el comportamiento de los individuos en la organización, su aproximación a estas variables es a través de las percepciones que los individuos tienen de ellas. Las variables consideradas en el concepto de clima organizacional son:

- Variables del ambiente físico, tales como espacio físico, condiciones de ruido, calor, contaminación, instalaciones, maquinas, etc. 
El comportamiento organizacional en su entorno hacia la actitud y conducta del ser humano para lograr ventaja competitiva en el desempeño laboral

- Variables estructurales, tales como tamaño de la organización, estructura formal, estilo de dirección, etc.

- Variables del ambiente social, tales como compañerismo, conflictos entre personas o entre departamentos, comunicaciones, etc.

- Variables personales, tales como aptitudes, actitudes, motivaciones, expectativas, etc.

- Variables propias del comportamiento organizacional, tales como productividad, ausentismo, rotación, satisfacción laboral, tensiones y stress, etc. (Torrecilla, 2005).

Esta relación que existe entre el clima laboral y la motivación afecta directamente a la competitividad de una empresa puesto que al mejorar el ambiente interno se fortalece el rendimiento de los empleados que puede observarse de una forma más clara en los procesos de calidad y elaboración de los productos y servicios, esto es de suma importancia en un mundo como el nuestro ya que se encuentra en constante movimiento debido a la globalización ${ }^{2}$. (Govea, Domínguez, \& San Agustín, 2012). Como se ha podido observar en las diferentes investigaciones hay una relación muy directa entre los climas organizacionales, las motivaciones laborales con la productividad de una organización $\mathrm{y}$ esto genera en el largo plazo una ventaja competitiva con aquellas organizaciones que no generan dentro de su clima organizacional ningún tipo de motivación o estímulo a sus trabajadores. Sin embargo hay metodologías dentro de las nuevas tendencias organizacionales y gestión administrativa que ayudan a los entornos empresariales a lograr una conexión más efectiva y directa con sus trabajadores, y esto es entendiendo cual es la mejor área en donde mejor puede desempeñarse un trabajador en base a sus capacidades (gestión por competencias y coaching). 
Los cambios a los que nos estamos enfrentando por la globalización y la alta exigencia del entorno en los últimos años y la situación actual nos hacen sin duda ir en búsqueda de herramientas gerenciales que coadyuven a gestionar y liderar nuestros recursos físicos e intelectuales de manera eficiente procurando con esto encontrar la luz que nos direccione permitiendo menguar nuestras posibles carencias (Lozano, 2008).

Las empresas que hayan aprendido a evaluar y a desarrollar las competencias de su personal estarán más capacitadas para afrontar los continuos retos del entorno. Por ello, se procura establecer opciones que fortalezcan el talento de cada miembro de una empresa, a fin de tener desempeños que se vean reflejados en el logro de objetivos eficientemente (Chávez, 2012).

Con base en esta idea surgen dos términos que deben ser analizados:

- La gestión por competencias, que busca aprovechar y moldear los conocimientos, habilidades, destrezas y actitudes de los individuos a fin de proporcionar el desempeño que persigue una organización competitiva.

- El ejercicio del coaching en la empresa, con el que de manera coordinada se puedan alinear las capacidades del personal con las expectativas de la organización a través de un mediador que funja como guía y orientador (Chávez, 2012).

Que se quiere indicar con esto, que las empresas deben tener dentro de sus filas personal altamente capacitado y que este alienado con la visión de la misma, esto con el fin de preservar un entorno competitivo de la organización dentro de su mercado y a su vez mantener esa conexión tan importante con el recurso humano que finalmente es que el enrumba la empresa al éxito. 
El comportamiento organizacional en su entorno hacia la actitud y conducta del ser humano para lograr ventaja competitiva en el desempeño laboral

Es así como el coaching se ha convertido en una herramienta que aporta y contribuye al desarrollo de estrategias que favorecen el crecimiento personal y profesional de quienes se esfuerzan por conseguir proactivamente el éxito; por lo tanto, este puede entenderse como un proceso integral que busca ayudar a las personas a producir resultados extraordinarios en sus vidas, carreras, negocios y organizaciones; a través del cual se mejora el desempeño, se profundiza en el conocimiento de sí mismos y mejora la calidad de vida, proporcionando un aprendizaje que genera transformación de comportamientos sostenidos en el tiempo, con acciones y reflexiones continuas (Lozano, 2008).

Podemos decir entonces, en primer lugar, que la adopción de modelos de perfeccionamiento administrativo en este momento obedece a exigencias que van más allá de las necesidades internas de una organización, que traspasan sus límites y que se enmarcan en un contexto global (Gomez, 2010). Es así, definitivamente la globalización es lo que mueve y determina todos los procesos, muchas organizaciones han cambiado su manera de administrarse y gerenciar obligados por los cambios generacionales y de tecnología que continuamente inundan nuestras sociedades, más que por un cambio o ganas de hacerlo por sí mismo.

\section{Materiales y métodos.}

La investigación está dentro de una metodología de tipo documental no experimental, ya que gran parte del contenido teórico esta soportado de páginas web, artículos de opinión, revistas digitales, sobre la temática de estudio. 


\section{Resultados.}

El indicador motivación donde se realizaron las siguientes preguntas: ¿La empresa le ayuda a desarrollar actitudes adecuadas para el desempeño de su tarea a través de talleres de motivación?, ¿Considera usted que el buen desempeño de los trabajadores radica en sentirse motivado por reconocimientos internos y satisfacción de necesidades? ¿Se encuentra motivado y con voluntad para cumplir con las responsabilidades asignadas dentro de la empresa? Los resultados determinaron un promedio positivo del $65 \%$ correspondiente a la alternativa "Siempre", lo cual indica que este indicador conforma una de las habilidades de la variable, la cual conlleva a un crecimiento personal y laboral de las personas (Quintero, Africano, \& Faría, 2008).

Un clima organizacional propicio incrementará las iniciativas para promover nuevos negocios, acometer nuevos proyectos y solucionar problemas; mejorará la comunicación interna; aumentará la competitividad y facilitará el gobierno de la organización. Pero, para esto se requiere:

- El fortalecimiento de las actividades de promoción de buena salud en la organización.

- $\quad$ El apoyo de la gerencia y la participación activa de los trabajadores, con sentido de pertenencia.

- $\quad$ El control de la presencia y el efecto de los factores de riesgo asociados con a accidentes y las enfermedades relacionadas con el trabajo.

- La motivación necesaria para dinamizar y garantizar el desarrollo de una política de higiene, seguridad, salud ocupacional y protección del medio. 
El comportamiento organizacional en su entorno hacia la actitud y conducta del ser humano para lograr ventaja competitiva en el desempeño laboral

- Un concepto global de desarrollo sostenible, calidad de vida, salud, seguridad en el trabajo y protección del medio en todos los nuevos proyectos que se pretendan emprender (Salazar, Guerrero, Machado, \& Cañedo, 2009)

- El entorno en el que vive y trabaja el hombre es un determinante de la productividad y calidad del trabajo que realiza. Las organizaciones inteligentes generan nuevos conocimientos a partir de su propio esfuerzo, pero para esto es premisa indispensable la creación de un clima organizacional favorable a la cooperación, que genera confianza y optimismo y destierre el egoísmo y las actitudes negativas que impiden el crecimiento tanto individual como corporativo (Salazar, Guerrero, Machado, \& Cañedo, 2009).

- La productividad de los empleados se ha medido de muchas formas. El indicador más sencillo son los ingresos por empleados, el cual representa la cantidad de resultado que cada empleado puede generar. A medida que los empleados y la organización se vuelven más eficaces en la venta de un mayor volumen y conjunto de productos y servicios con mayor valor añadido, deberían aumentar los ingresos por empleado (Torrecilla, 2005).

- El coaching es un proceso orientado a resultados y que tiene como consecuencia la mejora continua del desempeño, ya sea individual o grupal (Lozano, 2008).

\section{Conclusiones.}

Como conclusión general en base a todo lo que se ha abordado en la presente investigación se puede concluir la importancia que tiene la aplicación de estrategias corporativas que ayuden a mantener la competitividad de una organización. La aplicación de estrategia de coaching, gestión por competencias, y otras herramientas que ayuden a reorganizar la gestión de personal, procesos 
administrativos y gerenciales es lo que va a garantizar el éxito de toda la operatividad que motorizan a una empresa dentro de su mercado y en la sociedad. El capital humano es sumamente importante para lograr esto, ya que el trabajador es el que mueve la empresa y este debe ser seleccionado y estar capacitado para cada área que se le haya asignado para demostrar sus destrezas y habilidades (gestión por competencia) y para ello los gerentes deben convertirse en esos generadores y guías (coaching) de cambio que sigan estimulando ese dinamismo dentro del entorno organizacional para mantener la capacidad productiva, un capital humano armonizado y enraizado con la empresa y en fin una estructura corporativa alineada con la visión y misión de la misma.

\section{Otras conclusiones.}

Las definiciones de Competencia son innumerables. De ellas, citadas y reelaboradas por terceros autores se puede concluir lo siguiente:

1. Las competencias no se limitan al ámbito laboral. Pueden ser generalizables en otros contextos.

2. Tienen un significado exitoso, bien en el campo laboral, profesional o educativo.

3. Generalmente se asocian con actividades de tipo operativa en el ejercicio de una tarea o de un trabajo.

4. Para efectos administrativos se asocian con el rendimiento laboral

5. Pueden ser cuantificadas a través de escalas cada vez más complejas y sofisticadas

6. Están inmersas dentro de un modelo global. Esporádicamente se podrán identificar competencias individuales, pero sólo se acreditan en función de una tarea o de un objetivo práctico. 
El comportamiento organizacional en su entorno hacia la actitud y conducta del ser humano para lograr ventaja competitiva en el desempeño laboral

7. Tienden a permanecer con las personas. Son una especie de impronta (Gomez, 2010).

- Para que las organizaciones puedan lograr un alto grado de eficiencia es necesario trabajar en ambientes sumamente motivadores, participativos y con un personal altamente motivado e identificado con la organización, es por ello que el empleado debe ser considerado como un activo vital dentro de ella, por lo que los directivos deberán tener presente en todo momento la complejidad de la naturaleza humana para poder alcanzar índices de eficacia y de productividad elevada (Quintero, Africano, \& Faría, 2008).

- Para hablar de clima organizacional es imprescindible penetrar a fondo en la percepción de los trabajadores sobre las condiciones y procesos que se originan en el espacio laboral, así como en sus expectativas con respecto a la calidad de vida en el trabajo. Se trata de una mezcla de ciencia y artesanía. Es a la vez un área de acción social y de investigación científica. Trata de las personas y las organizaciones, de las personas en las organizaciones y de cómo funcionan. El clima organizacional se interesa en el cambio planificado; en lograr que los individuos, los equipos y las organizaciones funcionen mejor. El cambio implica sentido común; un trabajo arduo aplicado con diligencia a lo largo del tiempo; un enfoque sistemático orientado a metas, y un conocimiento sobre la dinámica de la persona, los grupos y la organización, del comportamiento de las personas y de los mismos procesos de cambio. El cambio se introduce mediante intervenciones, aún diagnósticas, y en actividades que estudian condiciones problemáticas específicas (Salazar, Guerrero, Machado, \& Cañedo, 2009).

- El Clima laboral se relaciona estrechamente con el comportamiento de los empleados así como en su conducta afectando directamente su comportamiento y por consecuencia su desempeño laboral (Torrecilla, 2005). 
- El desempeño laboral constituye un elemento fundamental para el funcionamiento de cualquier organización, por lo que debe prestársele especial atención dentro del proceso de administración de recursos humanos. La evaluación de dicho desempeño debe proporcionar beneficios a la organización y a las personas en virtud de contribuir a la satisfacción de los trabajadores en procura de garantizar el alcance de los objetivos institucionales (Pedraza, Amaya, \& Conde, 2010).

- Existen muchos motivos que impulsan la conducta de las personas, por lo tanto solo una compensación entendida desde una concepción integral, podrá satisfacer las distintas necesidades que energizan esos diversos motivos, influyendo así en el direccionamiento de la conducta, hacia lo que la organización necesita, lográndose una sintonía entre las metas de la organización y lo que sus empleados buscan (Bedodo \& Giglio, 2006).

- Las empresas deben favorecer la formación técnica que proporcione a los empleados habilidades que les permitan realizar una diversidad de tareas y posibiliten la rotación entre los diferentes puestos existentes y los nuevos que se puedan crear, al mismo tiempo que una formación más general que les permita conocer el funcionamiento del sistema, y asumir nuevas tareas que antes desarrollaban exclusivamente los supervisores y que pasan a ser responsabilidad de muchos (Fernández, 2002).

- Un colaborador que mejore sus habilidades, conocimientos y actitudes podrá desempeñar eficientemente sus actividades, además de lograr los objetivos organizacionales. La tarea de una empresa es saber gestionar por competencias, es decir, examinar el tipo de competencia adecuada para cada actividad asignada de trabajo y complementar la acción identificando los talentos y capacidades de cada uno de sus miembros para saber aprovecharlos u 
El comportamiento organizacional en su entorno hacia la actitud y conducta del ser humano para lograr ventaja competitiva en el desempeño laboral

orientarlos a fin de mejorar el nivel de competitividad y cubrir las necesidades de la empresa (Chávez, 2012).

- El coaching empresarial es una modalidad que va más allá de la orientación personal y de acciones ejecutivas, pretende enfocar esfuerzos que beneficien a la empresa, los grupos y los individuos, alineando sus expectativas de desarrollo, y acompañando a los protagonistas de la organización a mejorar su desempeño y a ser productivos. De esta forma, se deduce que las acciones del coaching empresarial están relacionadas en un sistema que comprenden elementos culturales y elementos relacionados con el comportamiento, las actitudes y las creencias de todos los que integran la empresa (Chávez, 2012).

- El coaching es un proceso que valora el bienestar y la autorrealización. Posee una doble vía de ser y hacer. Por el lado del ser hace referencia en el cuidarse a uno mismo, la calidad de vida y el crecimiento como seres humanos, mientras que por el lado del hacer es un procedimiento de fomentar los rendimientos y un estilo de liderazgo que obtiene buenos resultados desde cualquier ámbito en que se aplique el coaching. Es decir; el bienestar del individuo es primordial y el desarrollo humano es el método esencial de obtener los mejores resultados (Lozano, 2008).

\section{Recomendaciones.}

Como recomendación muy general es necesario que las empresas generen procesos internos que devengan en conocer a su trabajador, es decir, cuales son los problemas que estos enfrentan, que opinión tienen del manejo social de la empresa, que estímulos puede generar esta para que el trabajador se sienta parte de la organización. Hay que generar encuestas de opinión, matrices de 
resultados, análisis FODA, cualquier tipo de técnica que permita conocer al trabajador desde adentro y cuáles son sus perspectivas a futuro.

Es importante reconocer las aptitudes de los talentos humanos que motorizan a una organización, muchas veces un trabajador no rinde en su departamento sencillamente porque no tiene las cualidades para estar allí, por ello la visión de la gerencia de recursos humanos o en su defecto del jefe del departamento es aplicar todas las estrategias necesarias para conocer a su subordinado, ya que esto es lo que va a poder determinar para que área de la organización puede ser trasladado y de esta manera enfocar su máximo esfuerzo para los beneficios de ambas partes. Y esto es la gestión por competencias y si se requiere un proceso más profundo de determinación de competencias y cualidades es necesario que la organización contrate a los expertos en la materia para iniciar el proceso de coaching.

- Difundir políticas de desarrollo que le permitan al personal experimentar un progreso profesional e involucrarse con la organización. Las políticas deberán proyectarse por toda la empresa a fin de captar al personal para trabajar sobre la importancia del desarrollo personal y los climas de trabajo favorables como factor clave del éxito (Quintero, Africano, \& Faría, 2008).

- Diseñar programas de ascenso que proporcionen retos y competencia entre los trabajadores para que se esfuercen en realizar correctamente sus actividades y ser sobresalientes dentro de la institución, de esta manera se logrará un buen clima organizacional, un eficiente y eficaz desempeño laboral, además, de unos trabajadores satisfechos y realizados profesionalmente (Quintero, Africano, \& Faría, 2008).

- Es imprescindible implementar modelos para la evaluación del clima laboral dentro de las organizaciones puesto que gracias a esto es posible detectar las causas de las diversas 
El comportamiento organizacional en su entorno hacia la actitud y conducta del ser humano para lograr ventaja competitiva en el desempeño laboral

problemáticas internas y los motivos de la insatisfacción laboral, aunque no basta con reconocer dichas problemáticas si no que es necesario comenzar con planes de acción para revertir los inconvenientes y hacer que los empleados sientan que se encuentran en un buen ambiente organizacional, esto ayuda a la competitividad laboral ya que empleados satisfechos tienen un mayor desempeño y realizan trabajos de calidad, además de que disminuye el ausentismo, la rotación de personal y los empleados se sienten identificados con la empresa al verse involucrados en la toma de decisiones (Govea, Domínguez, \& San Agustín, 2012).

\section{Bibliografía.}

Bedodo, V., \& Giglio, C. (2006). Motivación laboral y compensaciones: una investigación de orientación teórica.

Chávez, N. (2012). La gestión por competencias y ejercicio del coaching empresarial, dos estrategias internas para la organización. Pensamiento \& gestión, 140-161.

Fernández, N. (2002). El desarrollo profesional de los trabajadores como ventaja competitiva de las empresas.

Gomez, L. (2010). Las competencias: Un modelo moderno de gerencia del talento humano. Revista Vanguardia Psicológica Clínica Teórica y Práctica, 14-32.

Govea, M., Domínguez, M., \& San Agustín, Y. (2012). Importancia del clima laboral en los resultados de una empresa y la competitividad. Contribuciones a la Economía.

Lozano, L. (2008). El coaching como estrategia para la formación de competencias profesionales. Revista Escuela de Administración de negocios.

Pedraza, E., Amaya, G., \& Conde, M. (2010). Desempeño laboral y estabilidad del personal administrativo contratado de la Facultad de Medicina de la Universidad del Zulia. Revista de Ciencias Sociales, 493-505. 
Quintero, N., Africano, N., \& Faría, E. (2008). Clima organizacional y desempeño laboral del personal empresa vigilantes asociados costa oriental del lago. Negotium.

Salazar, J., Guerrero, J., Machado, Y., \& Cañedo, R. (2009). Clima y cultura organizacional: dos componentes esenciales en la productividad laboral. Acimed, 67-75.

Torrecilla, O. D. (2005). Clima organizacional y su relación con la productividad laboral. Documento de Cátedra. Facultad deficiencias Políticas y sociales. 